\title{
Genetic Defects in SHROOM3 Lead to Congenital Heart Defects
}

\author{
Samuel Lorentz ${ }^{1}$, Matthew D Durbin, MD, $\mathrm{MS}^{2}$, Stephanie Ware, MD, $\mathrm{PhD}^{2,3}$ \\ ${ }^{1}$ Indiana University School of Medicine, ${ }^{2}$ Department of Pediatrics, Herman B \\ Wells Center for Pediatric Research, Indiana University School of Medicine, \\ Indianapolis, IN, ${ }^{3}$ Department of Medical and Molecular Genetics, Indiana \\ University School of Medicine, Indianapolis, IN
}

Background and Hypothesis: Congenital heart disease(CHD) is the most common birth defect, but most genetic contributors remain unknown. We recently identified CHD patients with variants in a gene called SHROOM3. The SHROOM3 protein impacts the actin cytoskeleton by binding ActinF and Rhokinase, causing actomyosin constriction. SHROOM3 also binds Dishevelled2(Dvl2), a component of Wnt/Planar cell polarity(PCP) signaling pathway, suggesting a connection between PCP signaling and actin-myosin contraction. We hypothesize SHROOM3 disruption alters PCP signaling and actin cytoskeleton during cardiac development, and is a novel contributor to CHD.

Project Methods: We analyzed the cardiac phenotype of Shroom3 gene trap knockout mice at embryonic day 14.5. We characterized the expression of Shroom3 during cardiac development using LacZ staining at important stages of cardiac development. Using IHC, we measured actomyosin disruption in Shroom3 knockout embryos. We preformed in silico analysis on previously identified SHROOM3 variants from patients with CHD.

Results: Shroom3 null mice had Ventricular Septal Defects $(0.73, p=0.0006)$, Double Outlet Right Ventricle $(0.33, p=0.04)$, Left Ventricular Noncompaction, and other CHD. Shroom3 mutant mice left ventricular wall thickness was $36 \%$ thinner compared to wild type mice $(99.0 \pm 8.6 \mu \mathrm{m}, 63.0 \pm 8.4 \mu \mathrm{m}, \mathrm{p}=0.005)$. LacZ shows the expression of Shroom 3 through important stages of cardiac development, and IHC shows actomyosin disruption. In silico analysis demonstrates CHD patients have SHROOM3 variants in highly conserved nucleic acid and protein sequences, and significant protein structural changes.

Conclusion and Potential Impact: Shroom3 null mice have cardiac defects resembling a Wnt/PCP disruption phenotype. Similarly, patients with CHD have likely pathogenic variants in SHROOM3. These data support a role for $S H R O O M 3$ in CHD pathogenesis and begin to elucidate mechanisms. Identifying SHROOM3's role in CHD is critical to understanding cardiac development as well as the diagnosis, management and treatment of $\mathrm{CHD}$. 